\title{
Survivable Timing Capability for a Worldwide Military Digital Communications System
}

\author{
HARRIS A. STOVER, SENIOR MEMBER, IEEE
}

\begin{abstract}
The evolving worldwide digital defense communications system (DCS) must be both more survivable and more responsive to the needs of its users than the analog system that it will replace. System timing problems not encountered in analog systems could prevent this. However, because of the availability of microprocessors, the survivability of system timing as a serious potential future problem for the DCS can be virtually eliminated; and it can be done economically. Important timing considerations for a wartime military digital communications system are discussed, and a description of a timing system that will economically satisfy survivability requirements is presented.
\end{abstract}

\section{INTRODUCTION}

A $\mathrm{T}$ present, the defense communications system (DCS) is predominantly an analog system employing digital transmission, i.e., those signals transmitted over digital transmission links are returned to analog form at most nodes through which they pass. When end-to-end digital signals are required, they are usually applied to modems that convert them to analog form for transmission over analog channels that might employ digital transmission links. This makes inefficient use of the facilities. Therefore, plans are developing for the network to become a digital network where signals enter the network in the form of bits and remain in that form until delivered to their destinations. This will permit more efficient use of the facilities, reduced operation and maintenance costs, more convenient use of encryption, reduction of interference and jamming, easier provision of new services, more effective system control, and many other advantages.

A worldwide synchronous switched digital communications network, in which time-slot interleaving is used for multiplexing and time-slot interchange is used for switching, has never existed, but the DCS is expected to become such a system. Then, a bit originating anywhere in the network must be available to fill its assigned time slot at every node through which it passes. Any two-bit streams originating in widely separated parts of the world must be properly interleaved in time wherever they share common facilities. Preferably, all originating signals should be syn-

Manuscript received July 16, 1984; revised April 15, 1985

The author is with the Defense Communications Agency, Washington, DC 20305. chronized within acceptable tolerances and remain synchronous through the network. To allow tolerances, variable storage buffers are used throughout the network to accommodate small clock errors as well as variations in signal transit times; but if these buffers overflow, traffic might be interrupted while several equipments at various locations are resynchronized in a particular sequential order. This could include spread-spectrum equipment used to overcome jamming, link encryption equipment, multiplexing equipment, switching equipment, and end-to-end encryption equipment in addition to various types of terminal equipment. The extensive use of such equipment and the importance of promptness in much military communication makes system timing much more demanding for military systems than civilian systems.

The digital DCS should be more responsive and provide greater communications survivability than the analog system being replaced. System timing problems could prevent this from happening. However, the microprocessor, if properly applied, will make possible the virtual elimination of survivability of system timing as a serious potential problem in the future digital DCS, and it can be done economically. The system described here is self-sufficient. Each major node has its own clock together with those alternates required for reliability. These clocks are coordinated with a timing reference distributed through the communications network. Propagation delays are automatically removed, and the master for the network and paths by which timing is distributed are automatically selected. Even the selection process is distributed throughout the network so that if massive destruction should occur, both the selection process and surviving portions of the timing system will still work properly. Timing references arriving over different paths are optimally combined. Timing changes made in any clock other than the master do not significantly affect any of the other clocks in the system. Provision is made for predicting and removing some errors during the very rare occasions when it is necessary for a clock to free-run. All of this is accomplished without the need for any node to communicate timing information with nodes farther away than immediate neighbors, and only a small amount of information need be exchanged with those neighbors. 


\section{IMPORTANT MILITARY CONSIDERATIONS}

Keep in mind the wartime need for survival of enough communications to make the most effective use of our military forces and their weaponry even in the face of a concerted enemy effort to disrupt communications. Further, recognize that the loss of timing can temporarily cause received information to be meaningless. Because of this, and the large number of complex synchronous equipments (encryption, spread-spectrum, etc.) used to counteract enemy efforts, a worldwide military digital communications system has system timing demands not likely to occur in a peacetime civilian system.

Each frame of a first-level digital multiplex in a U.S. civilian telephone system contains a single PCM sample of each of 24 voice channels. When timing slips occur because buffer capacity is exceeded, one frame is either deleted or repeated. Therefore, frame synchronization is not lost, but a single PCM sample, for signals that are sampled 8000 times/s, is either deleted or repeated [1]. Voice signals are usually very tolerant of such minor disturbances. However, things are not so simple in military digital systems where both link encryption and end-to-end encryption are used, where spread-spectrum coding is used to overcome jamming, where types of voice coding other than PCM are expected to be common place, and where a much higher percentage of the traffic can be expected to be interleaved digital traffic not carried on analog channels. In these systems, if buffer capacity is exceeded, resynchronization of several equipments in a particular sequential order is required.

In war, a military communications system can be expected to have massive destruction inflicted on many parts of the network over a short period of time. Because the location of the destruction cannot be predicted, every digital communications link must be capable of conveying timing information. Network synchronization at any node should be maintained so long as any link to the node remains operational, and maintaining accuracy for a considerable time after loss of the last link to the node can aid the rapid restoral of communications (particularly under enemy jamming conditions).

No facility should be particularly attractive for an enemy to attack. Therefore, system timing should not depend on the continued operation of any particular facility or group of facilities either internal or external to the network. (Items of this section are discussed more thoroughly in [2].)

Experience in synchronizing precise clocks for the defense satellite communications system (DSCS) has shown that system operators or technicians can cause serious problems unless they are provided very specific instructions by timing specialists before making any individual adjustment that could affect system timing. Similar experience has occurred in civilian networks where significant percentages of outages were traceable to activities of maintenance personnel [3]. It is impractical to have timing specialists available everywhere following massive wartime damage to many parts of a worldwide military network. Even if available, specialists would need access to information from computers in order to be most effective. Microcomputers should not only generate such information but also act on it, thereby providing a highly automated system that largely removes the need for manual participation.

Ideally, every network node should have a perfectly stable, perfectly accurate clock that is failure-free. We could not afford such clocks if they did exist. Fortunately, adequate stability and accuracy can be economically provided by making use of microcomputers and the transmission capabilities of the network, while failures can be accommodated by redundancy. The most stable and accurate time reference available to our military forces is Coordinated Universal Time as kept by the Naval Observatory, UTC(USNO). It should be used as a time reference for the network when available, but we must not be dependent on it. The DCS must always be capable of automatically selecting the best surviving clock in the network (or any fragmented portion of it) as the reference for the entire network (or any fragment of it), and that best network clock should normally be referenced to UTC (USNO). The DCS must also be capable of automatically determining the optimum paths for the distribution of the time reference.

\section{INFORMATION EXCHANGED BETWEEN NEIGHBORING NODES}

To provide the above capabilities every node has a reference clock which provides timing for all signals transmitted from the node. Clocks at all major nodes are each given a unique rank for establishing the order of ascension to master. The rank is assigned based on such things as accessibility of either direct or indirect timing references from the Naval Observatory, quality of the nodal clock, geographical location, and connectivity to other nodes. Needed capabilities can be provided without any node communicating timing information beyond its immediate neighbors, but each node provides the following information to neighboring nodes.

INFO 1: The rank of the network clock used as the ultimate master time reference for the local timing signal.

INFO 2: The least number of tandem links between the local node and its ultimate master.

INFO 3: The one-way transit time of the signal from transmitter to receiver when the time of transmission (e.g., of a particular sync pulse) is determined by the clock at the transmitting node and the time of reception is determined by the clock at the receiving node. (Ambiguities of less than $1 \mathrm{~min}$. can be removed by inverting the last sync pulse in every second except for the last second in every minute. It can be extended to a longer period by inverting the last pulse of the last second of the last minute of that longer period.)

INFO 4A: The measured but uncorrected error in the local clock based on information from those neighbors higher in the timing hierarchy than the local node. 
INFO 4B: The measured but uncorrected error in the local clock based on information from those neighbors at the same hierarchical level in addition to those higher.

INFO 5A: Estimated inaccuracy, stated as a variance, of INFO 4A based on information from all neighboring nodes higher in the timing hierarchy than the local node and the known characteristics of the types of transmission links to those nodes.

INFO 5B: Estimated inaccuracy, stated as a variance, of INFO 4B based on information from neighboring nodes at the same hierarchical level in addition to those higher in the hierarchy and the known characteristics of the types of transmission links to those nodes.

Assuming the above information is coded in 4 bit binary-coded decimals, an estimate of the required number of bits is

INFO 1: 1 million unique clock ranks 24 bits INFO 2: maximum of 1000 links to master

INFO 3: $1 \mathrm{~ns}$ resolution, $1 \mathrm{~min}$. range INFO 4A: $1 \mathrm{~ns}$ resolution, $1 \mathrm{~min}$. range 12 bits 44 bits INFO 4B: $1 \mathrm{~ns}$ resolution, $1 \mathrm{~min}$. range 44 bits INFO $5 A: 1 \mathrm{~ns}^{2}$ resolution, $10^{8} \mathrm{~ns}^{2}$ range INFO $5 B: 1 \mathrm{~ns}^{2}$ resolution, $10^{8} \mathrm{~ns}^{2}$ range TOTAL 44 bits 32 bits 32 bits 232 bits

Therefore, 232 bits/s are adequate to transmit all of the information once per second, and even double this number to provide error detection will only take $464 \mathrm{bits} / \mathrm{s}$.

\section{Automatic Local Determination of LeVel in TIMING HIERARCHY}

INFO 1 and INFO 2 automatically determine each node's level in the timing hierarchy. Every node initially entering the network is its own master (its ultimate network time reference) and informs its neighbors by giving the rank of its own clock as INFO 1 and zero as INFO 2. For each subsequent exchange of information, the microprocessor compares the rank of the local clock with INFO 1 received from each neighboring node. The highest rank is tentatively selected as the ultimate reference for the local clock; and, unless the local node has the highest rank, INFO 2 is compared to all those neighboring nodes having that highest rank for INFO 1. All neighboring nodes with the same highest rank for INFO 1 that also have the same shortest path for INFO 2 are tentatively selected as being one level higher in the hierarchy than the local node. If all neighboring nodes tentatively selected have supplied the same values of INFO 1 and INFO 2 for at least the last two information exchanges, the local node provides that same value of INFO 1 to all its neighbors and increases the corresponding minimum value of INFO 2 by 1 before supplying it to its neighbors. However, if any of the nodes tentatively selected has not provided the same values of INFO 1 and INFO 2 for at least the last two information

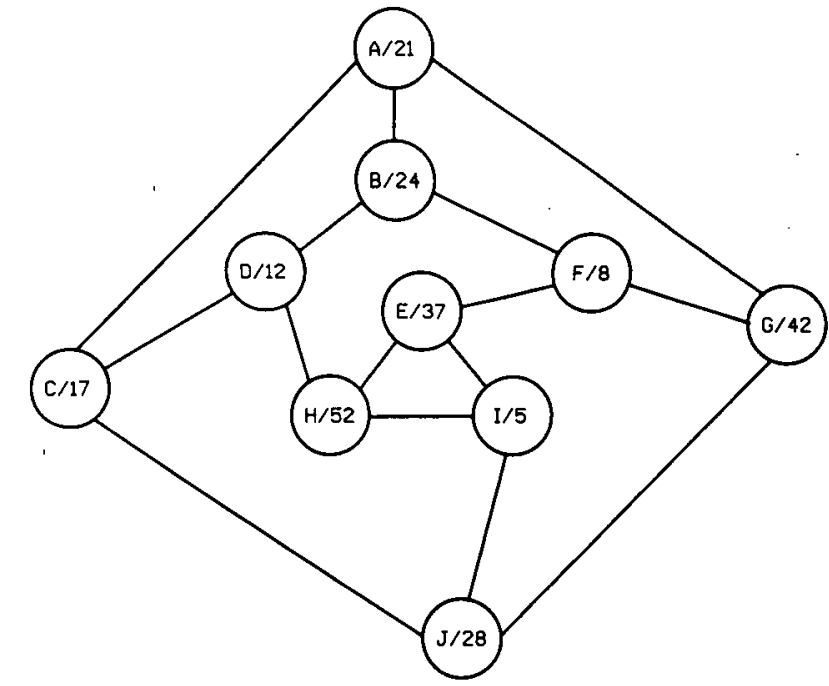

Fig. 1. A 10-node network used as an example of timing system selforganization.

exchanges, the local node rejects the tentative selection and provides its neighbors with the rank of its own clock as INFO 1 and zero as INFO 2. This procedure sweeps obsolete information from the network without being acted on whenever nodes or transmission links become inoperative.

Consider the 10-node network of Fig. 1, where the letters identify the nodes and the numbers provide their ranks. In Table I, the first column provides node identification and rank. The second column indicates connectivity by identifying neighboring nodes. The third column indicates the selection of nodes one level higher in the hierarchy, if any exist, or self-reference if they do not. The prime indicates that this is the first time that this information has been sent to neighbors. Since all clocks are initially entering the network, they all select self-reference in column 3. Column 4 shows the situation after the first information exchange. Since each node has received information only once from any of its neighbors, the tentative selections are rejected and all nodes self-reference again. In column 5, all information has been received twice so all tentative selections are accepted. This general procedure is followed through the remainder of the chart until the last column gives the resulting hierarchy which is shown in Fig. 2 . Note that this configuration, with every node in the network receiving the same information from its neighbors for the last two information exchanges, only required eight information exchanges. With one per second, only $8 \mathrm{~s}$ will fully organize the 10-node network. Since relatively stable clocks are loosely coupled, no significant changes occur during a period of organization or reorganization.

Table II shows what happens if link IJ is disabled. In this table, columns 1 and 2 are identical to Table I except the change is indicated. Column 3 of Table II is identical to the last column of Table I, and the change occurs before column 4 . Following the procedures described above, the 
TABLE I

Example of the INITIAL Self-Organization of THE Timing SYSTEM FOR THE NETWORK OF FIG. 1

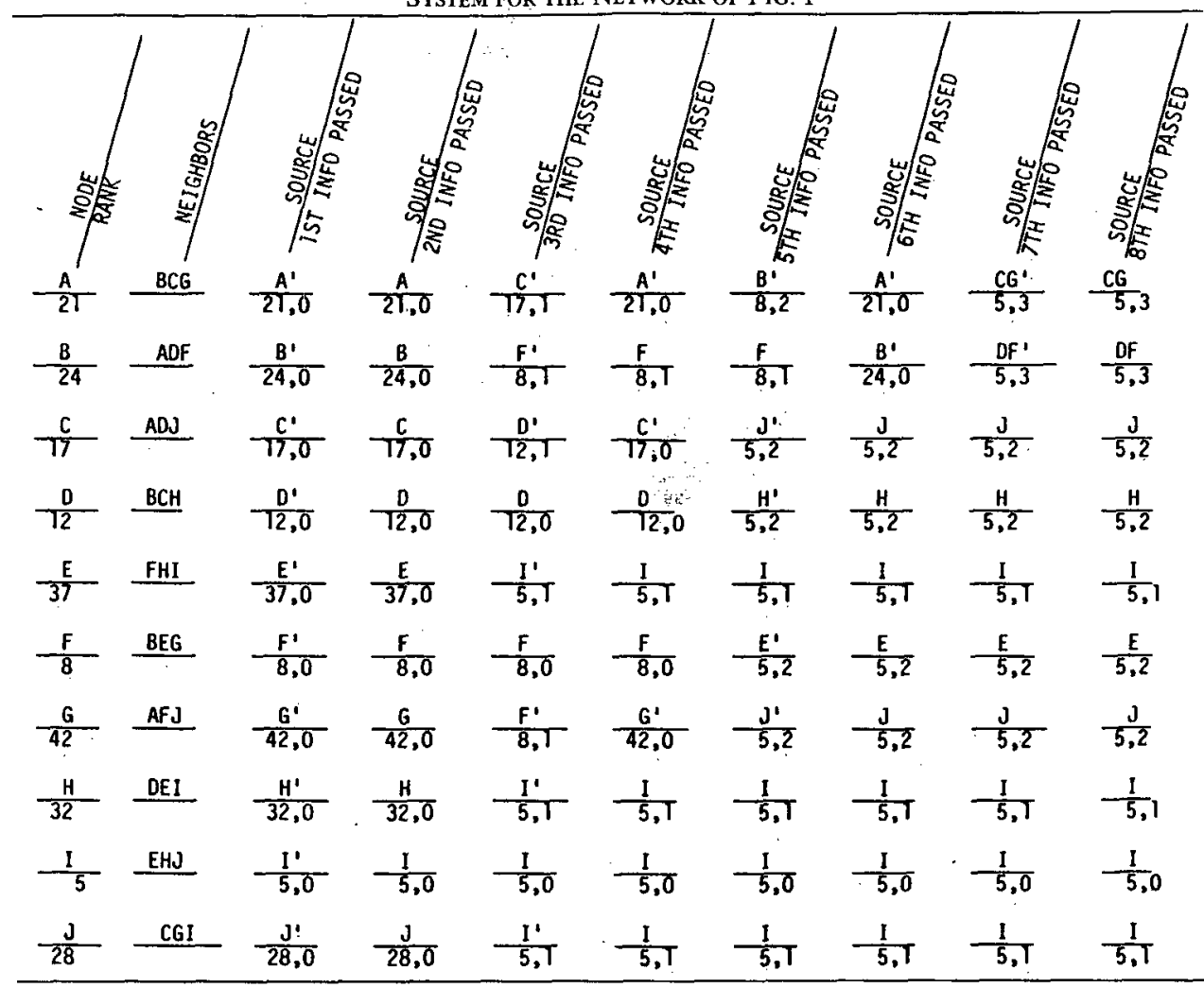

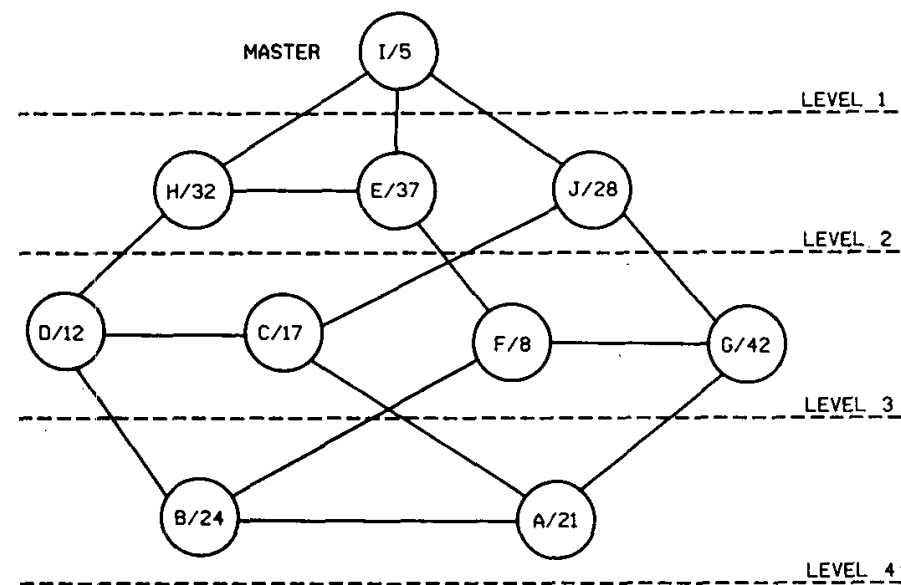

Fig. 2. The 10-node network of Fig. 1 following initial self-organization.

network is completely reorganized in five information exchange periods. The resulting hierarchy is shown in Fig. 3. Table III shows what happens if node I or all links connected to it are disabled. Columns 1 and 2 are identical to Table I except the change is indicated. Column 3 of Table III is identical to the last column of Table II, and the change occurs before column 4 . The network is completely reorganized in 11 information exchange periods, with node F moving from the third level to the top of the hierarchy. The resulting hierarchy is shown in Fig. 4. Note that for this particular configuration, in assuring that all obsolete
TABLE II

EXample of the Reoganization Of THE PReviously ORganized Timing System SHOWN IN Fig. 2 FOLLOWING FAILURE OF LINK IJ

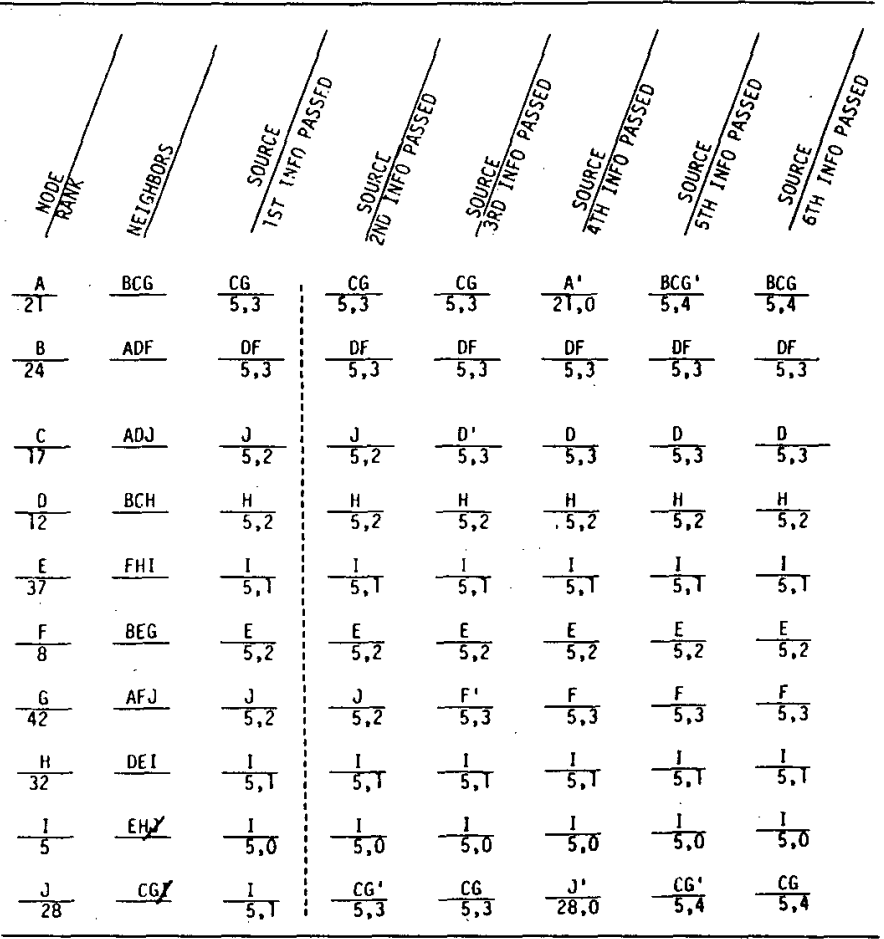

MITE: Dashed line Indicates roint in Sequence ithere Link IJ tlas Disabled 
TABLE III

Example of the Reorganization of the Previously

ORganized Timing System SHOWN IN Fig. 3 Following THE FaILURE OF NODE I

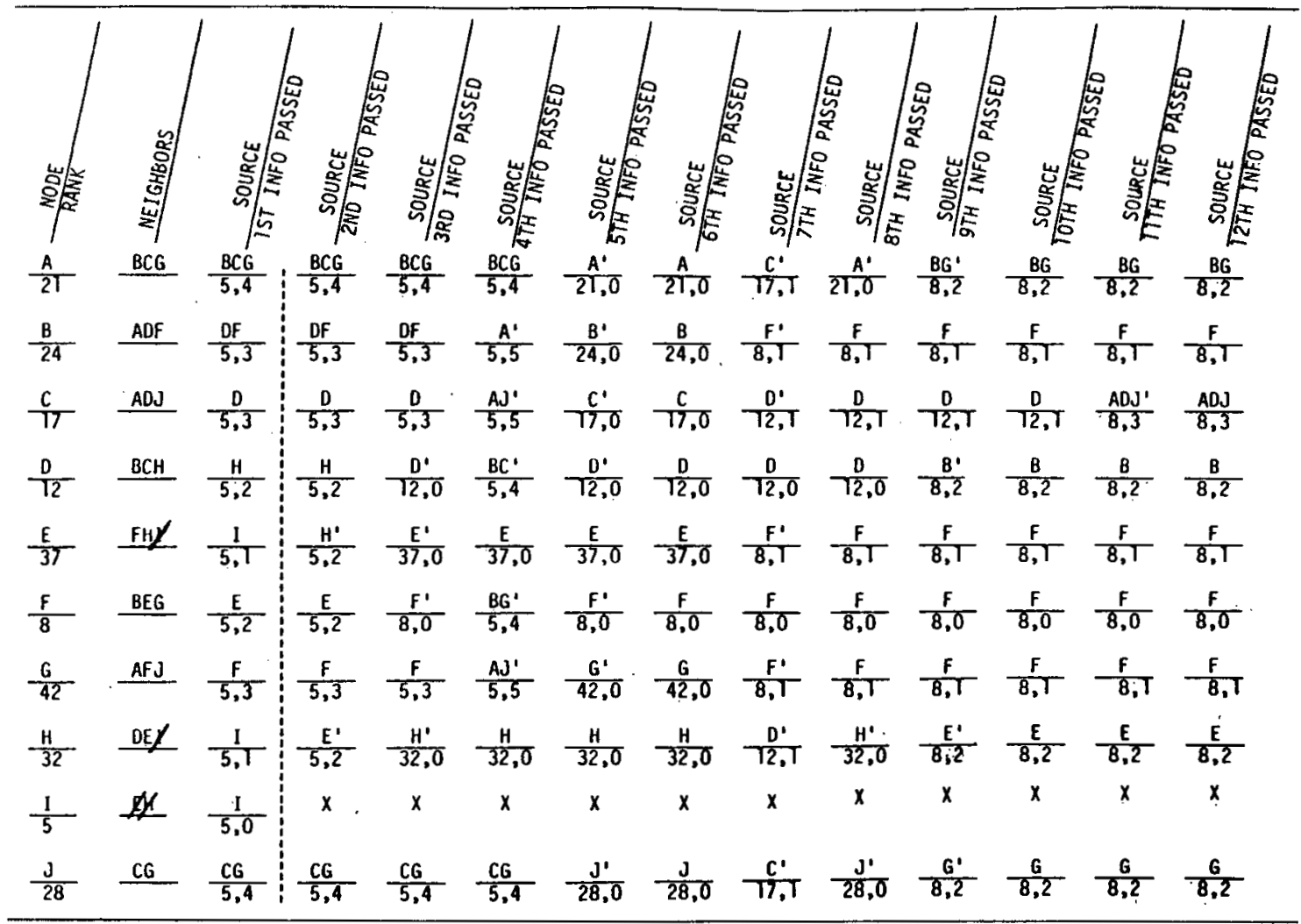

NOTE: Dashed Line indicates that links EI and HI were disabled at this point in the sequence

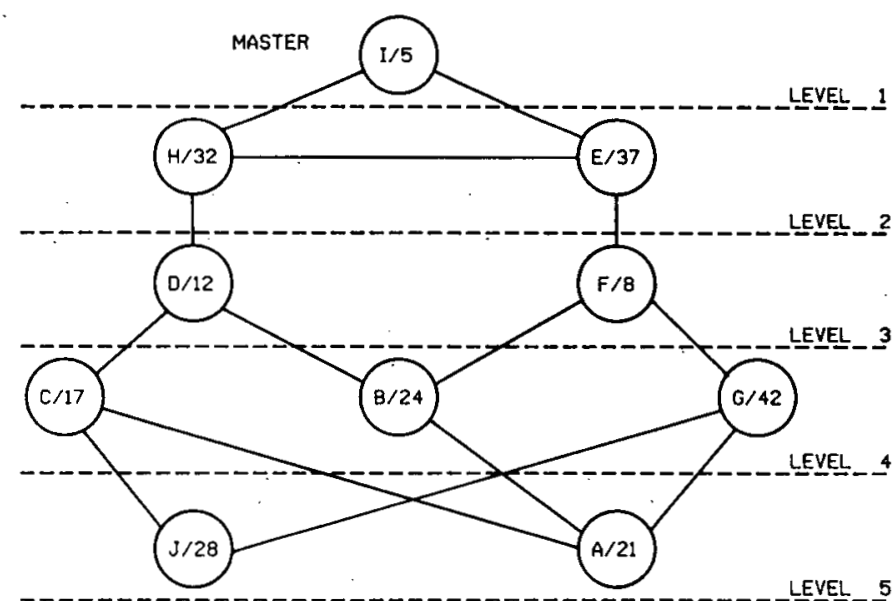

Fig. 3. The 10-node network of Fig. 2 following reorganization due to failure of link IJ.

information is removed from the network, all clocks are automatically returned to self-reference in columns 7 and 8 of Table III

\section{Comparison of Clock Time AT NEIGHBORING NODES}

INFO 3 is exchanged between neighboring nodes to compare their clock time. This comparison is shown in

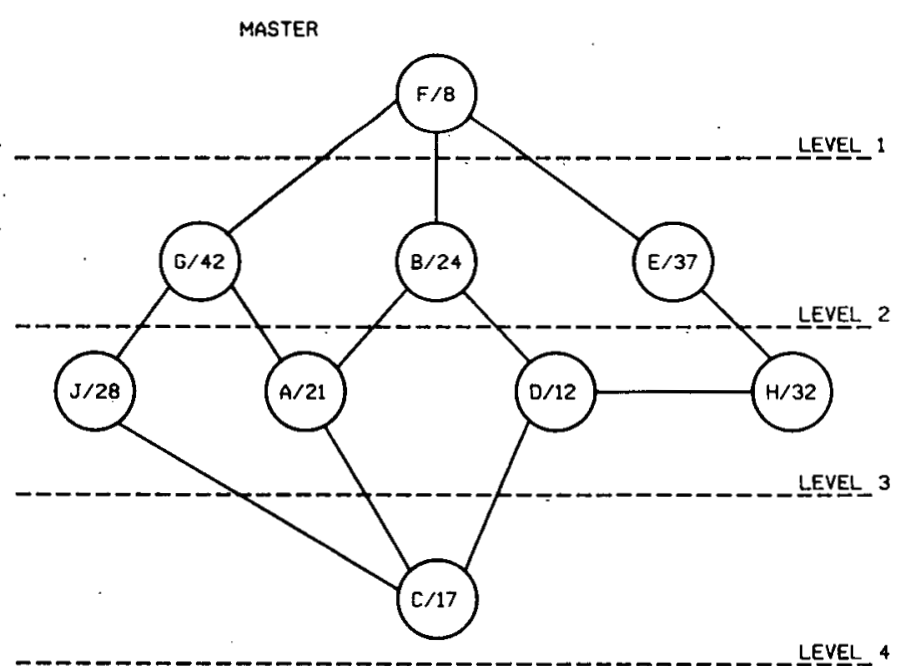

Fig. 4. The network of Fig. 3 following reorganization due to the failure of node I.

equation (1),

$$
T_{B}-T_{A}=\frac{\Delta_{B}-\Delta_{A}}{2}+\frac{D_{B A}-D_{A B}}{2},
$$

where $T_{B}-T_{A}$ represents the time difference between the two clocks; $D_{A B}$ represents the actual signal transit time from node A to node $\mathrm{B} ; D_{B A}$ represents the actual signal transit time from node $\mathrm{B}$ to node $\mathrm{A} ; \Delta_{A}$ represents the 
preliminary transit time measurement, INFO 3, as made at node $A$, i.e., $\Delta_{A}=T_{A}-\left(T_{B}-D_{B A}\right)$; and $\Delta_{B}$ represents the corresponding measurement at node $B$, i.e., $\Delta_{B}=T_{B}$ $\left(T_{A}-D_{A B}\right)$. If the actual signal transit time is the same in both directions, the last term of (1) cancels.

\section{Determining the ERror in the Local Clock}

If the local node is a master, i.e., INFO 1 is the rank of its own clock and INFO 2 is zero, and if it has an accurate reference either directly or indirectly to Naval Observatory time, UTC(USNO), then that reference is used to determine the measured but uncorrected error in the master clock. This is used for both INFO 4A and INFO 4B since there are no other nodes on the same level of the timing hierarchy with a master. Referencing the master to UTC(USNO) references the entire network to UTC(USNO) when it is fully organized and operating correctly. This is a reason for using the availability of a reference to UTC(USNO) in assigning ranks to network clocks at major nodes. To assure referencing UTC(USNO) for as long as it is available to the network, each clock should be assigned two ranks, one that is used when the node has an accurate external reference to UTC(USNO) and a lower rank for use when that reference to UTC is no longer available.

Each node receives error measurement information, INFO 4A and INFO 4B, from its neighbors. It adds the time difference between the clocks at each neighboring node and the local node as determined by (1), thereby providing an individual error measurement for its own clock based on each of its neighbors. Similarly, it receives corresponding error measurement accuracy information (expressed as a variance), INFO 5A and INFO 5B. It adds the accuracy information (variance). for the type of link connecting the two nodes. This provides the local node with accuracy information for each of the corresponding individual error measurements.

Equation (2) is used for combining clock-error measurement information from several neighbors [4],

$$
M_{c}=W_{1} M_{1}+W_{2} M_{2}+\cdots+W_{n} M_{n} .
$$

In (2), $M_{p}$ is a measurement value based on information from the $p$ th neighbor, while $W_{p}$ is a corresponding weighting factor as determined by (3),

$$
W_{p}=\frac{\frac{1}{\overline{\sigma_{P}^{2}}}}{\sum_{i=1}^{n} \frac{1}{\sigma_{i}^{2}}} .
$$

In (3), $\sigma_{p}^{2}$ is the accuracy information corresponding to the error measurement from the $p$ th neighbor, and $\sigma_{i}^{2}$ is the accuracy information corresponding to the error measurement from the $i$ th neighbor.

INFO 2 can be used by each node to determine its location in the timing hierarchy relative to its neighbors. If
INFO 2 provided by a neighbor is one less than INFO 2 for the local node, that neighbor is on the next higher level in the hierarchy. If it is the same, the neighbor is on the same level. If it is one more, the neighbor is on the next lower level.

To compute INFO $4 \mathrm{~A}$ at any node other than the master, only INFO 4B information from neighbors higher in the hierarchy than the local node (plus the measured time difference between the local clock and the clocks at these neighboring nodes) is used. If there is only one neighbor in the next higher level of the hierarchy, the error information based on INFO 4B from that single neighbor, as modified for the differences between clocks at the two nodes, is used as INFO 4A for the local node. If there is more than one neighbor in the next higher level, (2) is used to provide a weighted sum of the error information from them.

To compute INFO $4 \mathrm{~B}$ at any node other than the master, INFO 4A information from neighbors at the same level in the hierarchy is used in addition to INFO 4B information from nodes higher in the hierarchy. The use of either of two types of error measurement information, INFO 4A or INFO 4B, from neighbors at different levels permits time-error measurements from a larger number of neighbors to be used in computing INFO 4B while avoiding all closed feedback paths in the error information flow. This results in greater accuracy and stability.

To compute INFO 5A, accuracy information obtained by adding the accuracy for the links to neighbors higher in the hierarchy to the INFO 5B information received from those neighbors is combined by using (4),

$$
\sigma_{c}^{2}=\frac{1}{\sum_{i=1}^{n} \frac{1}{\sigma_{i}^{2}}}
$$

To compute INFO 5B, accuracy information based on INFO 5A from neighbors at the same level in the hierarchy and the types of transmission links to them is included in addition to accuracy information based on INFO 5B from neighbors higher in the hierarchy and the type of transmission links to them.

Note that the accuracy information, INFO 5A and INFO $5 \mathrm{~B}$, basically results from the inaccuracy (variance) of time-difference measurements made between the clocks at the ends of each transmission link. This variance is dependent on the type of transmission link used, and in some cases on its length also. Design engineers provide values to be used for each type of transmission link. Every time another transmission link is traversed, its variance is added so that the variance increases. However, additional parallel paths tend to reduce the variance. Equation (4) is not a precise evaluation, but it is a reasonable compromise for practical networks to avoid a requirement for any node to communicate timing information beyond its immediate neighbors. 


\section{Systematic Self-Monitoring}

Each node has clock-error information from each neighbor. Each of these has a computed variance associated with it. Based on these, each node derives a combined measurement of the error in its own clock along with an associated variance. The difference between this combined measurement and that based on each neighbor can be determined along with an associated variance. Provided the variances for the individual links have been well assigned, any of these computed differences that exceed the computed variance for that difference by more than a predetermined factor can be used to activate an alarm. This provides quantitative system monitoring based on the accuracy of different parts of the timing system.

The node serving as network master should reference its own clock and inform its neighbors that there are zero nodes between itself and its master. Each neighboring node connected to the master should be informing its neighbors that there is one link between it and its master. If the master detects that a neighbor persists in telling its neighbors otherwise, a problem has been detected. For every node in a stabilized timing system, either each neighboring node should be reporting the same number of links between itself and the master as the local node, or it should be reporting one more or one less than the local node. Any node consistently receiving reports from a neighbor that it is referencing the same highest ranking ultimate master, but that the number of links between it and the master is different from that of the local node by more than one link, has detected a problem.

\section{Minimizing the Propagation of Clock Errors TO OTHer Clocks}

Minimizing the effect that an error in any clock in the network can have on other clocks is important. Each node transmits its measured but uncorrected error to its neighbors. Each node also determines the time difference between its own clock and each neighbor's clock. This information for selected neighbors is combined to determine the local node's measured but uncorrected error. Because of the method used for this selection and computation, each node essentially determines its own error relative to UTC(USNO) if the master provides its neighbors with its own error relative to UTC(USNO), or relative to the master if the master has no way of measuring its own error relative to UTC(USNO). In making this computation, the new measured but uncorrected error for any node is based on the measured but uncorrected error received from selected neighbors during the previous information exchange period. Any clock-error changes that occurred since that previous information exchange period can temporarily introduce small errors.

This is illustrated in Table IV for the very simple case of three tandem links where node B computes its error based on information from node $A$, node $C$ computes its error based on information from node $\mathrm{B}$, and node $\mathrm{D}$ computes its error based on information from node C. Each computed error is the sum of the clock difference measured that period and the clock error provided by the other node during the previous period. Note that a permanent change in the clock at node $\mathrm{B}$, occurring between $N+1$ and $N+2$, introduced a temporary error in the clock-error measurement of node $\mathrm{C}$ that lasted only one information exchange period, after which the node $\mathrm{C}$ clock-error measurement was correct again. In the subsequent time period, the same size error occurred in the clock-error measurement at node $D$ and it also only lasted one exchange period. If the same amount of change should occur in clock B in each of $N$ consecutive exchange periods, the errors in the measurement of clock $\mathrm{C}$ would not accumulate but would remain constant for $N$ consecutive periods, after which they would be corrected. In this example, the total change in the clock at node B would have been $53 N$ units, but the resulting measurement error in node $\mathrm{C}$ would only be 53 units, lasting for $N$ time-exchange periods before returning to zero. In a normally operating system with good stable clocks, very loose coupling between clock-error measurements and correction of the clocks is desirable, i.e., it is desirable for each individual clock to approach a free-running condition while maintaining the long-term accuracy provided by the timing system. This will provide the maximum isolation from disturbances while still correcting the long-term inaccuracy and instability of the clocks. However, a capability must be provided for much tighter coupling to particular clocks under particular conditions, e.g., the clock of a new node entering the network. Provision must be made to minimize disturbances in other parts of the network when this more rapid correction capability is exercised. INFO 5A and INFO 5B should be adjusted to reduce the weighting for nodes making rapid changes in their clocks.

This feature can be explained in terms of a system with three levels of coupling plus a reset capability. If the measured but uncorrected error for the local node is less than $10 \mu \mathrm{s}$, the maximum rate of correction is limited to 1 ns every $10 \mathrm{~s}$. This permits a $10 \mu \mathrm{s}$ error to be corrected in slightly over a day with a resulting average temporary dynamic measurement error (due to clock corrections) of 100 ps during that correction period (negligible for our purposes). If the measured but uncorrected error is between $10 \mu \mathrm{s}$ and $100 \mu \mathrm{s}$, the maximum rate of correction is limited to $1 \mathrm{~ns} / \mathrm{s}$. This permits a $100 \mu \mathrm{s}$ error to be corrected in slightly over a day. If the measured but uncorrected error is between $100 \mu \mathrm{s}$ and $1 \mathrm{~ms}$, the maximum rate of correction is limited to $10 \mathrm{~ns} / \mathrm{s}$. This permits a $1 \mathrm{~ms}$ error to be corrected in a little over a day. If the error is over $1 \mathrm{~ms}$, a reset procedure is used in which a step phase correction is used to quickly correct this large error. In order to emphasize the use of information from nodes that generate minimum dynamic error, INFO $5 \mathrm{~A}$ and INFO 5B from nodes with errors less than $10 \mu \mathrm{s}$ follow the procedures discussed in the previous section. Those with errors between $10 \mu \mathrm{s}$ and $100 \mu \mathrm{s}$ add 10000 square ns to the normally determined values for INFO $5 \mathrm{~A}$ and INFO 
TABLE IV

EXample of The Dynamics OF THE Measured But

UNCORRECTED ERROR

\begin{tabular}{|c|c|c|c|c|c|}
\hline & \multicolumn{5}{|c|}{ INFORMATION EXCHANGE PERIOD } \\
\hline INFORMATION & $\mathrm{N}$ & $\mathrm{N}+1$ & $\mathrm{~N}+2$ & $\mathrm{~N}+3$ & $\mathrm{~N}+4$ \\
\hline $\begin{array}{l}\text { Measured But Uncorrected } \\
\text { Error in Clock A }\end{array}$ & +1740 & +1740 & +1740 & +1740 & +1740 \\
\hline $\begin{array}{l}\text { Time Difference }(B-A) \text { Between } \\
\text { Clocks } A \text { and } B\end{array}$ & -713 & -713 & -660 & -660 & -660 \\
\hline $\begin{array}{l}\text { Measured But Uncorrected } \\
\text { Error in Clock B }\end{array}$ & +1027 & +1027 & +1080 & +1080 & +1080 \\
\hline $\begin{array}{l}\text { Time Difference }(C-B) \text { Between } \\
\text { Clocks } B \text { and } C\end{array}$ & +920 & +920 & +867 & +867 & +867 \\
\hline $\begin{array}{l}\text { Measured But Uncorrected } \\
\text { Error in Clock C }\end{array}$ & +1947 & +1947 & +1894 & +1947 & +1947 \\
\hline $\begin{array}{l}\text { Time Difference }(D-C) \\
\text { Between Clocks } C \text { and } D\end{array}$ & -512 & -512 & -512 & -512 & -512 \\
\hline $\begin{array}{l}\text { Measured But Uncorrected } \\
\text { Errors in Clock D }\end{array}$ & +1435 & +1435 & +1435 & +1382 & +1435 \\
\hline
\end{tabular}

5B. Those with errors between $100 \mu \mathrm{s}$ and $1 \mathrm{~ms}$ add $4 \times 10^{6}$ square ns to the normally determined values for INFO $5 \mathrm{~A}$ and INFO 5B. Those clocks with measured but uncorrected errors greater than $1 \mathrm{~ms}$ transmit the maximum values for both INFO $5 \mathrm{~A}$ and INFO $5 \mathrm{~B}$ so that their neighbors will not use these clocks for reference information until they have been reset.

\section{Applying Clock-ERror Prediction}

High-quality clock oscillators are quite stable, but if they are adjusted this normal stability is disturbed. Therefore, timing adjustnents at a major node of the DCS should not be made directly to the clock oscillator, but should be made by adjusting the phase of the output of the oscillator. Digital control signals from the timing system microprocessor can control a high-resolution micro-phase-stepper to provide very accurate phase and frequency adjustments to the output of the nodal clock oscillator. The nodal clock signal should come from the output of the micro-phasestepper.

The timing system described earlier will provide a very accurate measurement of the error in the nodal clock signal (output of the micro-phase-stepper). Combining this with the known amount of correction by the micro-phase-shifter determines the accuracy of the undisturbed clock oscillator. When the timing system is working normally, i.e., a reference is available, control signals applied to the microphase-shifter slowly reduce the measured error in the nodal clock signal, and after a few days it is maintained it at a very low value. When the ability to measure the uncorrected error in a nodal clock signal is lost (e.g., all communications to the node are lost), a sequence of predictions of the errors that will occur during the free-running period can be used for corrections. These predictions can be based on observations of the clock oscillator errors during the time when measurements could be made. By providing a capability to predict and remove errors during a free-running period with sufficient accuracy for a long enough period of time, replacement of expensive cesium clocks with less expensive clocks is possible at many locations, and the performance of cesium clocks is also improved.

One method of predicting future clock errors based on past measurements employs the ARIMA (autoregressive integrated moving average) model [5], [6]. A basic equation for an $\operatorname{ARIMA}(0,3,1)$ model is

$y_{t+f}-3 y_{t+f-1}+3 y_{t+f-2}-y_{t+f-3}=a_{0} x_{t+f}+a_{1} x_{t+f-1}$.

In (5), $y$ represents a particular clock-error measurement or prediction at the instant of time indicated by the subscript, and $x$ represents a random phase disturbance at the instant of time indicated by the subscript. The $t$ in the subscript indicates the time interval of the last error measurement, and $f$ indicates time intervals into the future. During the calibration period, $t$ continually advances with each new error measurement; but during a prediction period, $t$ remains fixed and $f$ advances. Any term with a subscript greater than $t$ represents the future relative to the last measurement, and predicted values must be used for all such $y$ terms. There is no way of predicting future random disturbances so any $x$ terms with subscripts greater than $t$ must be assumed to be zero. The difference between each measurement and the prediction made just prior to the measurement is a random impulse, $x$, which is included in the actual measurement but could not be included in the prediction. Subtracting the predicted value obtained just prior to a measurement from each new measurement gives 
an inferred $x_{t}=y_{t}-\hat{y}_{t}$, where $\hat{y}_{t}$ represents a predicted value. The recursive process can be started at the initial measurement, $t=1$, by assuming that $x_{1}=0$, and that all measurements prior to that initial measurement are zero.

Using the above rules for determining when to use measured values, when to use predicted values, and when to assume that a quantity is zero, (6) and (7) are alternately applied during the calibration process

$$
\begin{aligned}
\hat{y}_{t+1} & =3 y_{t}-3 y_{t-1}+y_{t-2}+a_{1} x_{t} \\
x_{t} & =y_{t}-\hat{y}_{t} .
\end{aligned}
$$

First, (6) is used to generate a new estimate, $\hat{y}_{t+1}$, based on previous measurements. As soon as a new measurement $y_{t}$ is made, the predicted value, $\hat{y}_{t}$, is subtracted from it (7) to determine a value of $x_{t}$ to be used for the next recursive evaluation using (6).

After the process starts, the accuracy of each inferred $x_{t}$ increases, and therefore, so does the accuracy of the value of the initial estimate $y_{t+1}$ that would be used if prediction started at that point. Hence, if the calibration process has been proceeding for a long period of time before the prediction process begins, and the value of $a$ was selected to minimize the mean-square value of the $x$ 's, the prediction will begin with a very accurate value of $y_{t+1}$ in (8) when $f=1$

$$
y_{t+f}=3 y_{t+f-1}-3 y_{t+f-2}+y_{t+f-3}+a_{1} x_{t+f-1} .
$$

Using (8) repeatedly, increasing $f$ by one each time, after a few cycles only past predicted values will be used for determining each new predicted value.

Because high-quality stable clocks change very slowly, computations do not have to be made very frequently. It is probably desirable to average measurements taken once per second for several minutes or even longer before applying them to these equations in order to reduce the effects of measurement noise.

Another technique for such prediction is the use of the Kalman filter.

\section{CONCLUSIONS}

Employing microprocessors to virtually eliminate a serious potential survivability problem of the future digital DCS, i.e., that of survivability of system timing, is technically and economically feasible as well as strategically prudent.

\section{REFERENCES}

[1] J. E. Abate et al., "The switched digital network plan," Bell Syst. Tech. J., vol. 56, no. 7, pp. 1297-1320, Sept. 1977.

[2] H. A. Stover, Attributes for Timing in a Digital DCS, Defense Communications Engineering Center, Eng. Pub. no. 1-80, DTIC AD-A093647, July 1980.

[3] R. L. Mitchell, "Survey of timing/synchronization of operating wideband digital communications networks," in Proc. 10th Annu. Precise Time and Time Interval (PTTI) Applications and Planning Meeting, Goddard Space Flight Center, Nov. 1978, pp. 405-435.

[4] H. A. Stover, "Network timing/synchronization for defense communications," IEEE Trans. Commun., vol. COM-28, pp. 1234-1243, Aug. 1980. (This reference contains a list of 62 additional references that might be of interest to the reader.)

[5] H. A. Stover, "Application of clock error prediction for digital communications," in Proc. MILCOM '83, Washington, DC, Oct. 31-Nov. 2, 1983, pp. 731-737.

[6] G. Box and G. Jenkins, Time Series Analysis: Forecasting and Control. San Fransico, CA: Holden-Day, 1970.

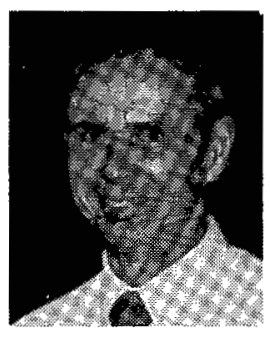

Harris A. Stover (S'49-A'51-M'56-SM'63) was born in Conrad, IA. He received the B.S.E.E. degree from Iowa State University, Ames, in 1950, and the M.S.E.E. and Ph.D. degrees from the University of Iowa, Iowa City, in 1957 and 1959, respectively.

He worked for the Collins Radio Company, Cedar Rapids, IA, from 1950 to 1971, where he participated in a wide range of engineering activities. Since 1972, he has worked for the Defense Communications Agency, most of the time working with transmission systems. He is presently working on communications system architecture.

Dr. Stover has been granted 43 U.S. patents, and is a member of Eta Kappa Nu, Phi Eta Sigma, Sigma Xi, and Phi Kappa Phi. 